\title{
TÉCNICA DE SANDWICH CERRADO EN RESTAURACIONES DIRECTAS CON RESINA COMPUESTA EN PIEZAS DENTARIAS POSTERIORES. REPORTE DE CASO
}

\section{Closed SANDWich TeCHNiQUe in DiRect RESTORATIONS With COMPOSITES IN POSTERIOR TEETH. CASE REPORT}

\author{
César Lamas Lara* \\ Giselle Angulo de la Vega*
}

\section{RESUMEN}

\begin{abstract}
T os protocolos restauradores en odontología varían con la finalidad de obtener resulLtados más predecibles y estables en el tiempo. Este artículo detalla un protocolo de aplicación de un adhesivo autograbante como agente de enlace y la aplicación de una resina compuesta mediante la técnica incremental oblicua en cavidades con ionómero de vidrio (la técnica de sándwich) para la resolución de un caso clínico. Los resultados fueron satisfactorios para el paciente ya que restauró adecuadamente la pieza dentaria y se le devolvió la estética y la función. En conclusión, el conocimiento de los materiales así como las técnicas optimizan los resultados y los vuelve más predecibles y estables en el tiempo.
\end{abstract}

Palabras clave: caries dental, adhesivos, resinas compuestas

\section{ABSTRACT}

The restoratives protocols in Dentistry are changing over the time with the purpose of obtain more predictable and stable results in the time. In the present article there is detailed a protocol of application of one self-adhesive as agent of link and the application of a composite resin using the incremental oblique technology in cavities with glass ionomer (sandwich technique) in the resolution of a clinical case. Results: The results were satisfactory for the patient since it was possible to restore adequately the dental piece

* Cirujano dentista. Docente de la Universidad San Martín de Porres, filial norte. .

* Cirujano dentista, con estudios en la especialidad en Rehabilitación Oral en la Universidad Nacional Mayor de San Marcos. 
involved, returning the aesthetics and the lost function. Conclusions: The knowledge of the materials to use as well as the technologies are going to lead to optimizing the results turning these more predictable and stable in the time.

Keywords: dental caries, adhesives, composites resins

\section{INTRODUCCIÓN}

Los avances tecnológicos en odontología conllevan a la aparición constante de materiales nuevos así como nuevas técnicas, pero muchas con poco aval científico y técnico. El profesional se ve envuelto, más que en avances, en tendencias impuestas por las casas comerciales y por tanto obtiene resultados poco satisfactorios y de corta duración.

Existen diferentes protocolos restauradores en relación con las resinas compuestas basados en principios de simplicidad y practicidad para el profesional y que pueden lograr resultados óptimos con estos materiales (Albers, 2002; Conceição et al., 2005 y Ardu et al., 2006).

Cabe destacar que no todas las resinas compuestas se comportan de la misma manera, tanto por sus propiedades físicas como estéticas. Una restauración visualmente correcta resulta insuficiente si no cumple su función dentro del sistema estomatognático (Lamas et al., 2009 y Lamas et al., 2011).

En la actualidad, estas se pueden clasificar de acuerdo con las estrategias de unión a los estratos dentarios; de este modo, se clasifican en adhesivos convencionales (con la aplicación de ácido grabador), adhesivos autograbadores y materiales autoadhesivos (Van Meerbeek et al, 2011; Van Meerbeek et al., 2010 y Lamas et al., 2011).

En los adhesivos autograbantes, se recomienda la aplicación de ácido fosfórico al 37 \% por quince segundos sobre la superficie del esmalte para que se pueda garantizar un sellado marginal altamente confiable en las restauraciones (Padrós-Serrat et al., 2003 y Soares et al., 2007).

El objetivo del caso fue devolver al paciente una adecuada estética y función masticatoria mediante el uso de materiales restauradores directos aplicando una técnica sencilla de resultados predecibles.

\section{REPORTE DEL CASO}

Una paciente de 24 años acude a la consulta clínica para la restauración de sus piezas dentarias. Al examinar a la paciente, se aprecia el desprendimiento de una restauración a nivel de la pieza 3.6 (figura 1).

\section{DIAGNÓSTICO}

Del estado de salud general: la paciente goza de un buen estado de salud general, es decir, sin riesgo sistémico al tratamiento estomatológico. 
Del estado de salud estomatológico: la paciente sufre el desprendimiento de la restauración en la pieza $3.6(\mathrm{O})$.

\section{PROCEDIMIENTO CLÍNICO}

Examinando las necesidades de cada cavidad, se decide utilizar como base cavitaria ionómero de vidrio modificado con resina (figura 2).

Una vez verificada la cavidad, se procede a aplicar el ácido fosfórico en gel al 37\% solo sobre el esmalte por quince segundos (figura 3). Se lava con abundante agua y se seca con papel absorbente.

Posteriormente, se aplica ionómero de vidrio modificado con resina como base cavitaria debido a la profundidad (figura 4) y se fotopolimeriza por veinte segundos. Al aplicar ionómero de vidrio después del grabado con ácido fosfórico, se evita hidrolizar y deteriorar la superficie.

Inmediatamente, se aplican dos capas de un sistema adhesivo de séptima generación en la cavidad oclusal y se fotopolimeriza por diez segundos según las indicaciones del fabricante (figura 5).

Luego de la aplicación del adhesivo, se procede a aplicar los incrementos de resina compuesta utilizando la técnica incremental oblicua (figuras 6, 7 y 8). Los incrementos de resina compuesta se aplican colocando incrementos en cada cúspide sin contacto entre cada incremento; y se fotopolimeriza por veinte segundos. Posteriormente, se completa cada cúspide para otorgar la anatomía respectiva fotopolimerizando cada incremento por viente segundos. Finalmente, se realiza el control de la oclusión y el acabado y pulido con cauchos y gomas abrasivas (figuras 9 y 10).

Figura 1: vista inicial

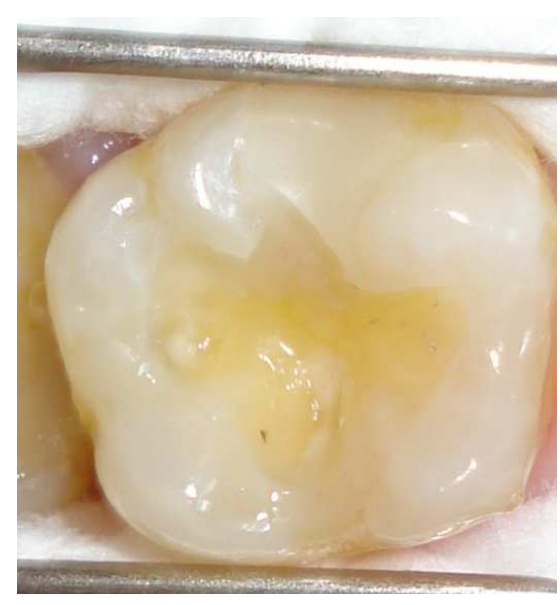


Figura 2: aislamiento absoluto y medición de la profundidad de la cavidad

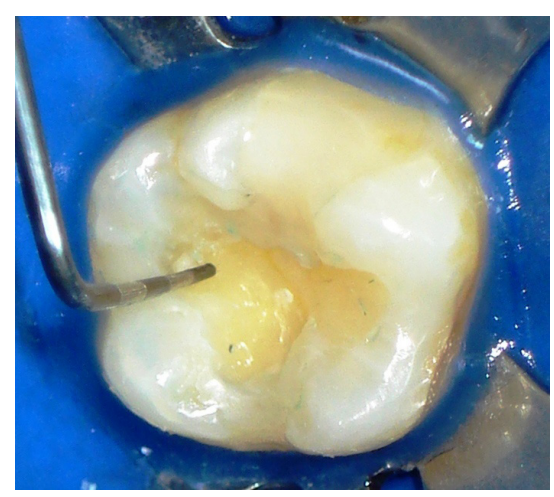

Figura 3: aplicación del ácido fosfórico en esmalte

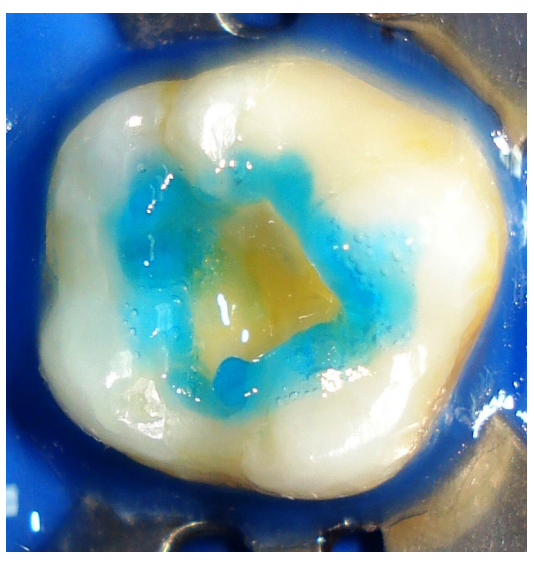

Figura 4: aplicación de ionómero de base

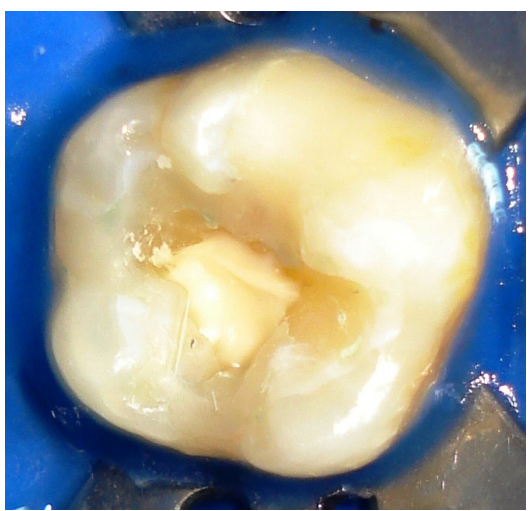


Figura 5: aplicación de adhesivo de séptima generación

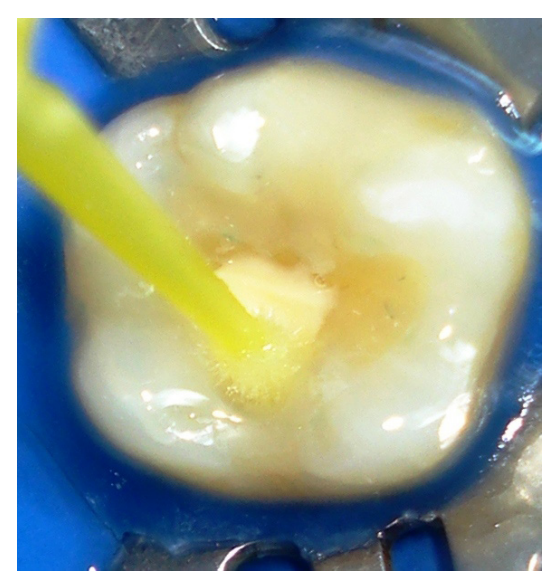

Figura 6: aplicación de incrementos de resina compuesta

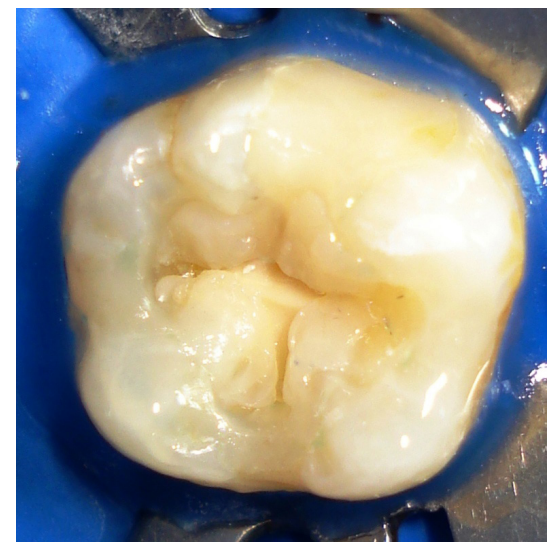

Figura 7: aplicación del incremento de resina compuesta en la cúspide distolingual

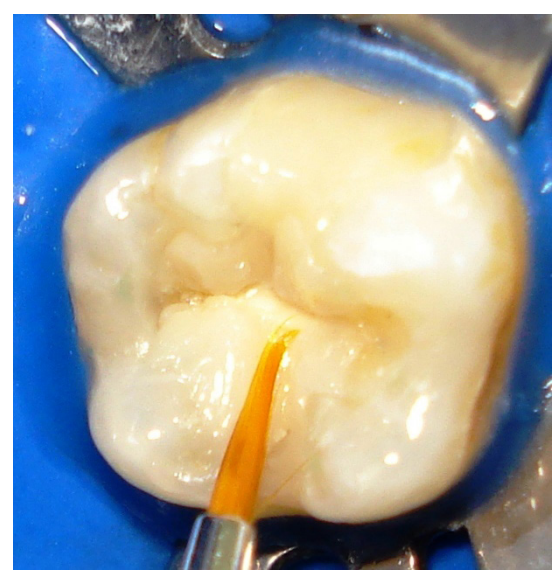


Figura 8: aplicación de los incrementos de resina compuesta en la cúspide distovestivular

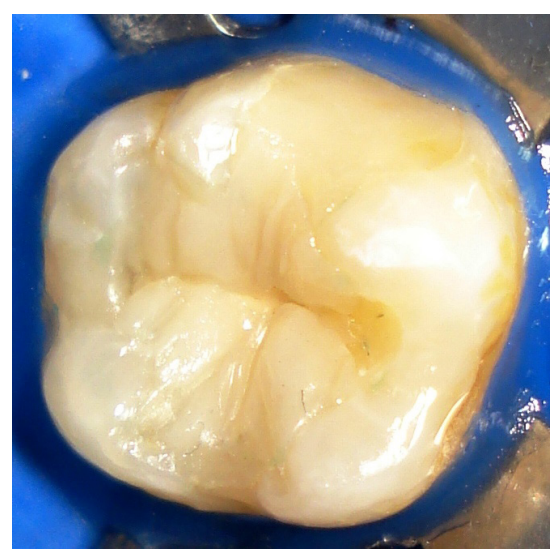

Figura 9: término de la aplicación de los incrementos de resina compuesta

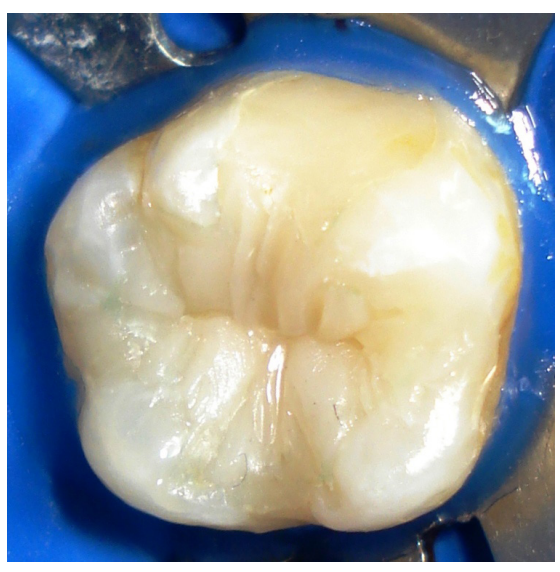

Figura 10: vista final de la restauración

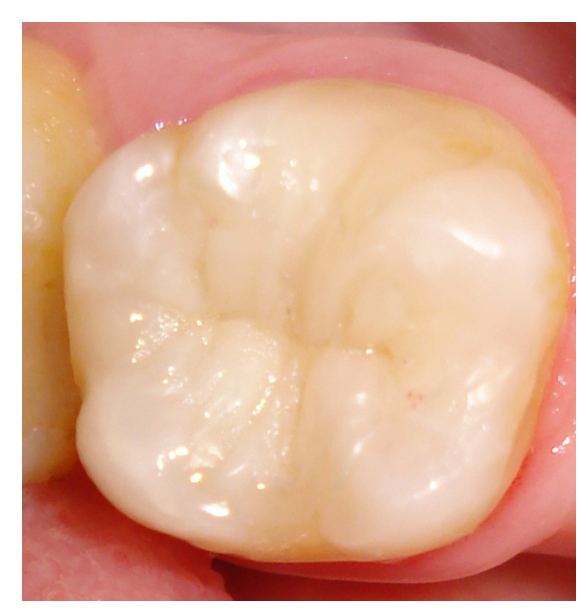




\section{RESULTADOS}

Los resultados fueron satisfactorios para el paciente ya que se restauró adecuadamente la pieza dentaria involucrada y le devolvió la estética y la función.

Si bien los adhesivos autograbantes aparecieron en el mercado con la finalidad de simplificar los pasos clínicos, en la práctica esta simplificación no es tan real, pero sí garantizan protocolos más seguros para los procedimientos.

\section{DISCUSIÓN}

La aplicación de adhesivos autograbantes se populariza cada vez más y esto se debe a la practicidad de la aplicación; sin embargo, aplicar ácido grabador con estos adhesivos parece una sugerencia válida, al menos en esmalte, para mejorar la interfase de unión resina-dentina (Van Landuyt et al., 2006 y Miguez et al., 2003).

La técnica incremental oblicua en la aplicación de resinas compuestas tiene el objetivo de reducir el estrés de contracción del material y obtener un valor de 0.5, aceptable para el sector posterior. Otra alternativa es reducir el volumen de la resina compuesta empleando materiales como los ionómeros de vidrio, a menor volumen menor contracción. Estos métodos para reducir el estrés no son excluyentes entre sí (Davidson, 1994; Feilzer et al., 1988 y Lamas et al., 2013).

\section{REFERENCIAS BIBLIOGRÁFICAS}

Albers, H. (2002). Tooth colored restorations: principles and techniques (9th edition). Ontario, Canada: BC Decker Publishing. 203.

Ardu, S. y Krejci, I. (2006). Biomimetic direct composite stratification technique for the restoration of anterior teeth. Quintessence Inter 37 (3), 167-74.

Conceição, E. et al. (2005). Restaurações Estéticas. Compósitos, Cerâmicas e Implantes. Porto Alegre, Brasil: Ed Artmed. 152.

Davidson, C. (1994). Glass ionomer bases under posterior composites. Journal of Aesthetic Dentistry 6, 223-226.

Feilzer, A.; De Gee, A. y Davidson, C. (1988). Curing contraction of composites and glass-ionomer cements. J Prosth. Dent 59 (3), 297-300.

Lamas, C. y Angulo G. (2013). Restauraciones estéticas en piezas posteriores aplicando la técnica incremental oblicua y adhesivos de quinta generación. Reporte de caso. In Crescendo 4 (2), 349-56. 
Lamas, C. y Angulo, G. (2009). Reconstrucción del sector anterior con resinas compuestas. Odontología Sanmarquina 12 (2), 90-92.

Lamas, C. y Angulo, G. (2011). Técnica de mock up y reconstrucción por capas anatómicas. Odontología Sanmarquina 14 (1), 19-21.

Lamas, C.; Angulo, G. y Alvarado, S. (2013). Aplicación de un adhesivo autograbante y uso de la técnica incremental oblicua para resinas compuestas en restauraciones directas en el sector posterior. Reporte de caso. Odontología Sanmarquina 16 (1), 40-43.

Míguez, P.; Castro, P.; Nunes, M.; Walter, R. y Pereira, P. (2003). Effect of Acid etching on the enamel bond of two self etching systems. Journal of Adhesive Dentistry 5, 107-112.

Padrós-Serrat, J.; Monterrubio-Berga, M. y Padrós-Cruz, E. (2003). Adhesivos autograbantes: ¿Grabar o no grabar? RCOE Ago 8 (4), 363-375.

Soares, C.; Guimarães, C.; Freitas, P. y Soares, A. (2007). Effects of previous treatments on bond strength of two self-etching adhesive systems to dental substrate. Journal of Adhesive Dentistry 9, 291-296.

Van Landuyt, K.; Kanumilli; De Munck, J.; Peumans, M.; Lambretchs, P. y Van Meerbeek, B. (2006). Bond strenght of a mild self etchself-etch adhesive with and without prior acid etching. Journal of Dentistry 34, 77-85

Van Meerbeek, B.; Peumans, M.; Poitevin, A.; Mine, A.; Van Ende, A.; Neves, A. et al. (2010). Relationship between bond-strength tests and clinical outcomes. Dent Mater 26 (2), e100-21.

Van Meerbeek, B.; Vargas, M.; Inoue, S.; Yoshida, Y.; Peumans, M.; Lambrechts, P. et al. (2001). Adhesives and cements to promote preservation dentistry. Oper Dent 26 (6), $119-44$. 